\title{
The differential anti-tumour effects of zoledronic acid in breast cancer - evidence for a role of the activin signaling pathway
}

\author{
Caroline Wilson ${ }^{1 *}$, Penelope Ottewell ${ }^{2}$, Robert E Coleman ${ }^{1}$ and Ingunn Holen ${ }^{1}$
}

\begin{abstract}
Background: Neo-adjuvant breast cancer clinical trials of zoledronic acid (ZOL) have shown that patients with oestrogen negative (ER-ve) tumours have improved disease outcomes. We investigated the molecular mechanism behind this differential anti-tumour effect according to ER status, hypothesising it may in part be mediated via the activin signaling pathway.

Methods: The effects of activin A, its inhibitor follistatin and zoledronic acid on proliferation of breast cancer cells was evaluated using either an MTS proliferation assay or trypan blue. Secretion of activin A and follistatin in conditioned medium (CM) from MDA-MB-231, MDA-MB-436, MCF7 and T47D cell lines were measured using specific ELISAs. The effects of ZOL on phosphorylation domains of Smad2 (pSmad2c + pSmad2L) were evaluated using immunofluorescence. Changes seen in vitro were confirmed in a ZOL treated subcutaneous ER-ve MDA-MB-436 xenograft model.

Results: Activin A inhibits proliferation of both ER-ve and oestrogen positive (ER + ve) breast cancer cells, an effect impaired by follistatin. ZOL significantly inhibits proliferation and the secretion of follistatin from ER-ve cells only, which increases the biological activity of the canonical activin A pathway by significantly increasing intracellular pSmad2c and decreasing nuclear accumulation of pSmad2L. In vivo, ZOL significantly decreases follistatin and pSmad2L expression in ER-ve subcutaneous xenografts compared to saline treated control animals.

Conclusions: This is the first report showing a differential effect of $Z O L$, according to ER status, on the activin pathway and its inhibitors in vitro and in vivo. These data suggest a potential molecular mechanism contributing to the differential anti-tumour effects reported from clinical trials and requires further evaluation in clinical samples.
\end{abstract}

Keywords: Breast cancer, Zoledronic acid, Activin, Follistatin, Phosphorylated Smad2

\section{Background}

The addition of ZOL to neo-adjuvant chemotherapy has been shown to enhance the response of invasive breast cancer to chemotherapy [1]. However, not all breast tumours are equally responsive to the drug, with some studies suggesting that ZOL has a greater effect on primary tumour response and disease recurrence in patients with ER-ve, as opposed to ER + ve, tumours [2,3]. In vitro, ZOL inhibits proliferation and induces apoptosis of the ER-ve cell line MDA-MB-231, an effect not seen in the ER + ve cell line MCF7 [4]. The anti-tumour effects of

\footnotetext{
* Correspondence: c.wilson@sheffield.ac.uk

${ }^{1}$ Academic Unit of Clinical Oncology, University of Sheffield, Medical School, Sheffield, UK

Full list of author information is available at the end of the article
}

ZOL reported from in vitro studies include reduced adhesion, migration and invasion of tumour cells, mediated by inhibition of farnesyl diphosphate (FPP) synthase and reduced prenylation of small GTPases (enzymes that hydrolyze guanosine triphosphate) [5].

The clinical neo-adjuvant breast cancer study, ANZAC, evaluated the biological effects of addition of ZOL to first cycle of $\mathrm{FEC}_{100}$ chemotherapy, and showed serum levels of follistatin significantly decreased following administration of ZOL in postmenopausal women [6]. Furthermore the addition of ZOL to chemotherapy reduced serum follistatin levels at day 5 post treatment specifically in patients with ER-ve tumours compared to patients receiving chemotherapy alone [7]. This may reflect a fall in the 
secretion of follistatin from ER-ve breast tumours that is not seen in ER + ve tumours.

Follistatin is a paracrine antagonist of activin and both proteins modify breast cancer cell proliferation. Activin is produced by breast cancer cells, inhibiting their proliferation, while follistatin binds to activin and prevents receptor binding with the type II receptor (ActRII), thus promoting proliferation [8]. Once activin binds to ActRII, dimerization occurs with ActRIB and the receptor associated intracellular proteins $\operatorname{Smad} 2$ and 3 are phosphorylated (Figure 1) [9]. Smad phosphorylation occurs either at the $\mathrm{C}$ terminus or at a linker region joining the $\mathrm{MH} 1$ and $\mathrm{MH} 2$ domains, with different effector functions; the $\mathrm{C}$ terminus being a tumour suppressor and the linker region being a tumour promoter [10] (Figure 1). ER-ve breast cancer cell lines have been shown to be insensitive to the anti-proliferative effects of activin [11], however this effect does not appear to be due to low expression of the activin type II receptor, with evidence that MDA-MB-231 express activin type II receptors [11] and MDA-MB-436 have a functional activin-signaling pathway showing phosphorylation of Smad2 in response to exogenous activin following removal of follistatin from the medium [12]. These data indicate that exogenous neutralisers of activin, i.e. follistatin, are responsible for the lack of inhibition of proliferation in response to activin in ER-ve cell lines, rather than absence of/non functional activin receptors.
We provide the first evidence that ZOL can affect the activin signaling pathway specifically in ER-ve breast cancer cell lines by a dual mechanism; decreasing secretion of follistatin and preventing nuclear localization of linker phosphorylated Smad2.

\section{Methods}

\section{Cell lines and reagents}

ER-ve (MDA-MB-231, MDA-MB-436) and ER + ve (MCF7, T47D) human breast cancer cells were purchased from European Collection of Cell Lines and routinely cultured in RPMI $+10 \%$ foetal calf serum (FCS). Evaluation of secretion of proteins from cell lines into conditioned medium (CM) and effects on $\mathrm{pSmad} 2 \mathrm{C}$ was performed using human activin $A$ and follistatin quantikine ELISAs and the cell based phospho-Smad2/3 fluorescent ELISA, purchased from R\&D systems (Oxford, UK). Cell titre 96 Aqueous One solution cell proliferation assay (MTS) was purchased from Promega (Southampton, UK). The tumour samples were obtained from MDA-MB-436 previously described xenograft studies [13]. Recombinant human activin A and follistatin were purchased from R\&D systems (Oxford, UK). ZOL ([(1-hydroxy-2-(1H-imidazol-1-yl) ethylidene $]$ bisphosphonic acid) was supplied as the hydrated disodium salt by Norvartis Pharma (Basel, Switzerland). Primary antibodies were purchased from Santa Cruz USA (Rap1a), Abcam UK (GAPDH) and Cell Signaling UK

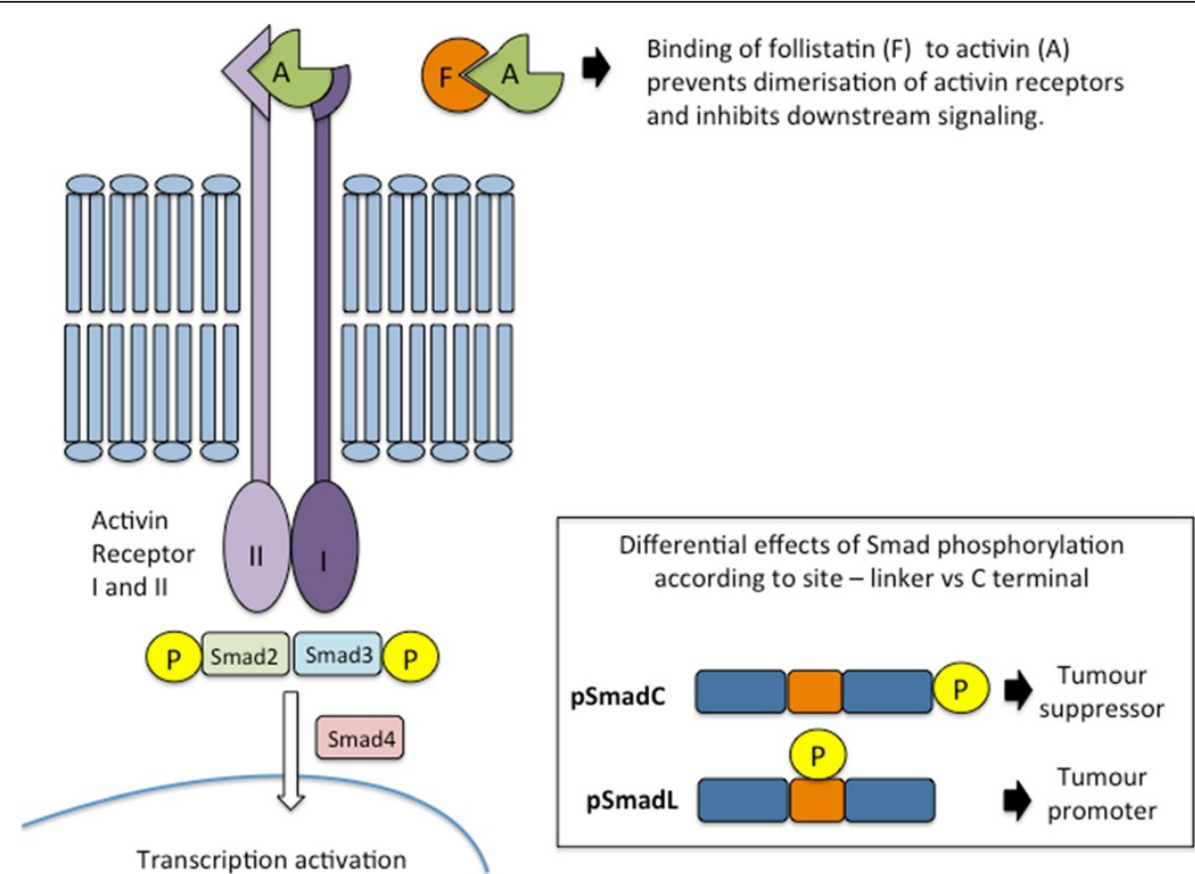

Figure 1 The canonical activin pathway. Activin binds to activin type II receptors resulting in phosphorylation of the C terminus of Smad2 (pSmad2C) or smad3 followed by nuclear translocation with co-receptor Smad4. Follistatin binds to activin preventing binding the type II receptor. Phosphorylation at the linker region of Smad2 or smad3 occurs downstream of cytoplasmic proteins such as RAS and nuclear proteins such as cyclin dependent kinases. The effector function of phosphorylated Smad2 is dependent on the site of phosphorylation; C terminus phosphorylation resulting in tumour growth suppression and linker phosphorylation resulting in tumour growth promotion. 
(phosphoSmad2, all secondary antibodies). SB-431-542 was purchased from Tocris bioscience (Bristol, UK).

\section{Western blotting}

Cells were lysed in cell lysis buffer (Sigma-Aldrich) and proteins were resolved using 12\% SDS-PAGE. Proteins were immobilized on polyvinylidene difluoride (PVDF) membrane, blocked (5\% milk) and probed with antibodies specific to unprenylated Rap1a (1:200), pSmad2L (1:1000), with GAPDH (1:20,000). Representative blots from three separate experiments are shown.

\section{Enzyme linked immunoabsorbance assays}

Human Follistatin and Activin A ELISAs were carried out according to the manufacturers instructions using CM from tumour cells. Minimum detection limits were $29 \mathrm{pg} / \mathrm{ml}$ and $3.67 \mathrm{pg} / \mathrm{ml}$, respectively, with intra-assay CVs $<15 \%$. Molar ratios were calculated as follows; mean $\mathrm{CM}$ concentrations ( $\mathrm{pmol} / \mathrm{L}$ ) divided by molecular weight of the protein, expressed as a ratio (follistatin:activin).

The quantification of total smad $2 / 3$ to phosphoSmad2/3 was carried out using a cell based phospho-Smad2/3 fluorescent ELISA. 1.5x10 ${ }^{3}$ MDA-MB-231 cells were seeded in a 96 well plate and the ELISA was carried out according to the manufacturers instructions.

\section{Proliferation assay}

Cell proliferation was assessed either using an MTS assay or viable cell counting with trypan blue. For the MTS assay $1.5 \times 10^{3}$ MDA-MB-231/3×10 $0^{3}$ MCF7 cells were seeded in 96 well plates and for the trypan blue assay $1 \times 10^{5}$ cells were seeded in 6 well plates. Cells were serum starved for 24 hours before addition of recombinant protein/drug in RPMI $+10 \% \mathrm{FCS}$, cells were washed and protein/drug replaced every 24 hours. At completion of the MTS assay $20 \mu \mathrm{l}$ of MTS solution was added directly to each well and quantified on a plate reader. At completion of the trypan blue assay cells were trypsinised to remove from wells and trypan added in a 50:50 concentration of cell suspension to trypan blue and counted using a haemocytometer.

\section{Immunofluorescence}

To visualize pSmad2C and pSmad2L, $2 \times 10^{4}$ MDA-MB$231 / 4 \times 10^{4}$ MCF7 cells were seeded in chamber slides, serum starved for 24 hours and then treated for 48 hours with ZOL $(50 \mu \mathrm{M})$. Cells were fixed ( $4 \%$ paraformaldehyde) and blocked (5\% goat serum) prior to incubation with phosphoSmad2 antibodies (1:100). After incubation with secondary antibodies (1:100) and fluorescein-avidin, coverslips were mounted with DAPI and viewed on an inverted fluorescent light microscope. Images of $\geq 100$ cells per chamber manually scored for nuclear localization using the blue dapi counter stain as a nuclear localiser.

\section{Immunohistochemical staining for follistatin and pSmad2L}

Paraffin embedded tumour sections from previously published in vivo experiments were used [13]. The in vivo experimental design used female MF1 nude mice injected with $5 \times 10^{5}$ MDA-MB-436 cells sub-cutaneous and animals were treated weekly with $100 \mu \mathrm{g} / \mathrm{kg}$ intra peritoneal ZOL vs. saline control for 6 weeks starting on day 7. Tumours were processed for histology using standard protocols. Sections were dewaxed, blocked (3\% $\mathrm{H}_{2} \mathrm{O}_{2}$ in methanol) for 10 minutes followed by trypsin antigen retrieval for 15 minutes at $37^{\circ} \mathrm{C}$. Further blocking ( $5 \%$ goat serum $/ 1 \%$ bovine serum albumin) for 30 minutes was followed by addition of primary antibody overnight (pSmad2L 1:100/follistatin 1:200). Secondary antibodies were added (1:200) for 30 minutes followed by a 6 -minute incubation with DAB. All animal experiments were carried out in accordance with local guidelines and with Home Office approval under project license 40/2343 held by Professor N. J. Brown, University of Sheffield, UK.

\section{Statistical analysis}

Unless stated otherwise, all experiments were carried out with 3 replicates and 3 repeats. Prism GraphPad (5.0a) was used for statistical analysis. Data analysis was by nonparametric Mann-Whitney test to compare differences between groups or Wilcoxon Signed-Rank test to compare related groups. Data represent mean and SEM. Statistical significance is defined as a $\mathrm{p}$ value $=<0.05$.

\section{Results}

\section{Activin A inhibits the proliferation of ER + ve and ER-ve} breast cancer cells

Previous reports have shown that proliferation of ER + ve cells is inhibited by activin [14], but the effect of activin on proliferation of ER-ve cell lines is less clear [11]. In order to compare the effect of activin and follistatin on proliferation in ER-ve and ER + ve cell lines, a time course and dose response MTS assay was performed. Both the ER-ve (MDA-MB-231) and ER + ve (MCF7) cells showed a significant decrease in proliferation compared to control following addition of activin $\mathrm{A}$ on days 1 and 3 (Figure $2 \mathrm{~A}+\mathrm{B}$ ) which was lost by day 5 (data not shown). There was a significant dose-dependent inhibition of proliferation with increasing doses of activin $\mathrm{A}$ in both cell lines. These results show that both ER-ve and ER + ve cell lines are responsive to the growth inhibitory effect of activin A. To confirm the decrease in proliferation was occurring in an activin dependent manner in ER-ve cell lines given the controversy in the literature, MDA-MB-231 cells were treated with activin $6000 \mathrm{pg} / \mathrm{ml}+/-$ an ALK4/5 inhibitor SB-431-542 (10 $\mu \mathrm{M} / \mathrm{l})$ for 72 hours. The ALK4/5 inhibitor will prevent ActRII dimerising with its type I receptor 


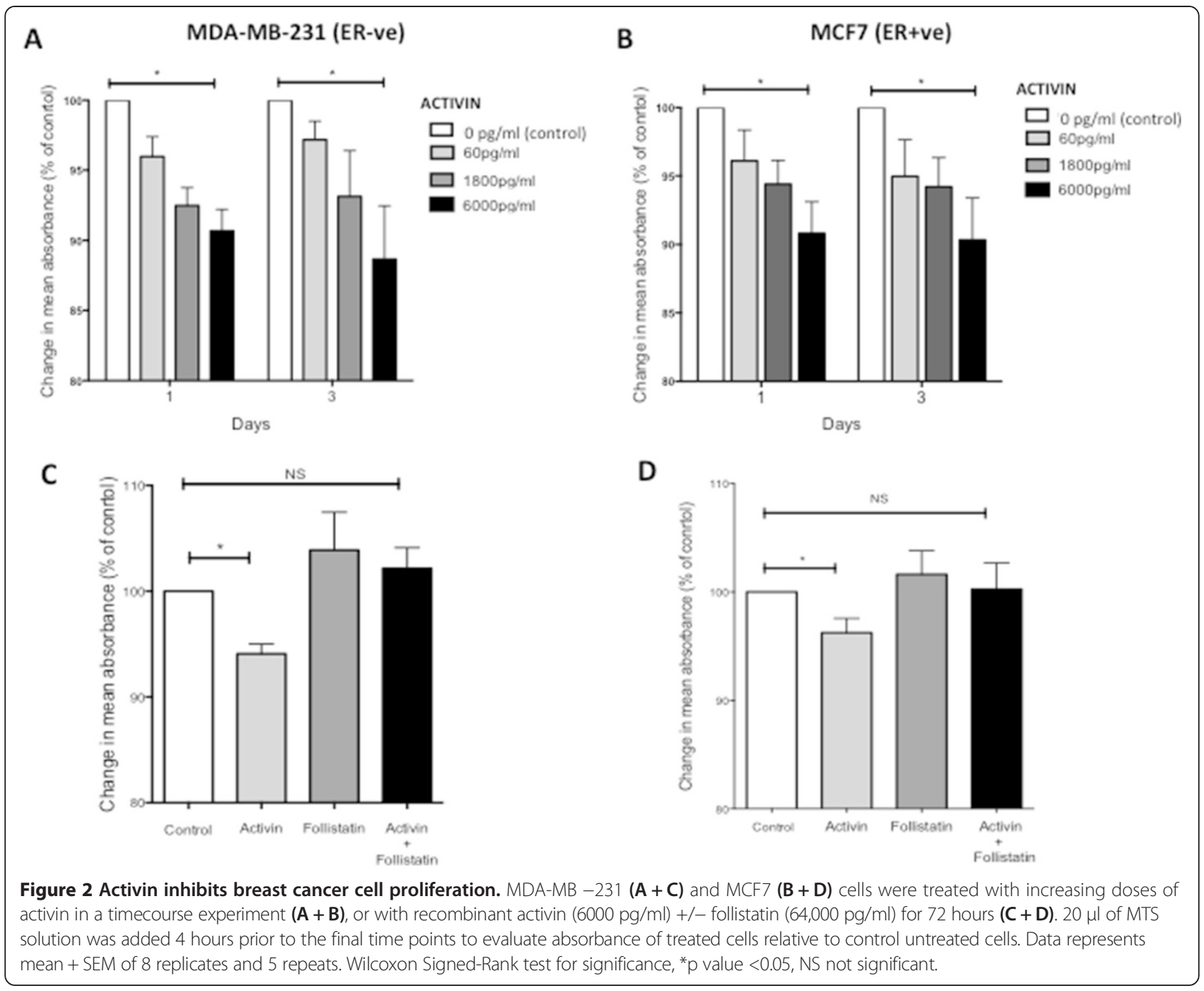

ALK4. MDA-MB-231 cells showed less inhibition of proliferation with addition of the ALK4/5 inhibitor to activin (mean \% change in absorbance from control Activin; -11\%, Activin + ALK4/5 inhibitor -3.4\%) confirming the changes in proliferation in ER-ve cells was activin-dependent (data not shown).

\section{Follistatin impairs the inhibition of proliferation induced} by activin $A$

Follistatin is reported to negate the anti-proliferative effect of activin [12]. In order to evaluate the effect of follistatin in ER-ve (MDA-MB-231) and ER + ve (MCF7) cells they were treated with $6000 \mathrm{pg} / \mathrm{ml}$ of activin A in the presence or absence of follistatin $(64,000 \mathrm{pg} / \mathrm{ml})$ for 72 hours (activin concentrations were chosen to replicate inter-tumoural levels of activin in breast tumours [15]). The significant inhibitory effect of activin A on cell proliferation was negated in the presence of follistatin in MDA-MB-231 cells (mean \% change from control; Activin $-6 \%$ [SEM 0.92], Activin + follistatin $+2.6 \%$ [SEM
1.9]). In MCF7 cells a similar, but non-significant, trend was also seen (mean \% change from control; Activin -3.2\% [SEM 1.3], Activin + follistatin -0.02\% [SEM 2.4]) (Figure 2C+D). These data provided further indication that ER-ve cell lines are sensitive to the growth inhibitory effects of activin $\mathrm{A}$ and that this effect is inhibited by follistatin.

\section{ER-ve cells secrete more activin A and follistatin than ER +} ve cells

The quantity of activin A and follistatin secreted from ER-ve (MDA-MB-231 and MDA-MB-436), and ER + ve (MCF7 and T47D) cells was determined by ELISA. As shown in Figure 3A, both ER-ve cell lines secreted significant levels of activin A (MDA-MB-231 $=561 \mathrm{pg} / \mathrm{ml}$ [SEM 104.9] $\mathrm{p}$ value $=0.0034, \mathrm{MDA}-\mathrm{MB}-436=430 \mathrm{pg} / \mathrm{ml}$ [SEM 73.8] $\mathrm{p}$ value $=0.0436)$. Follistatin was detectable in the medium from all cell lines, although the levels were much lower in ER + ve cell lines (mean level pg/ml; MCF7 $=200, T 47 D=108)$ compared to ER-ve cell lines 
A

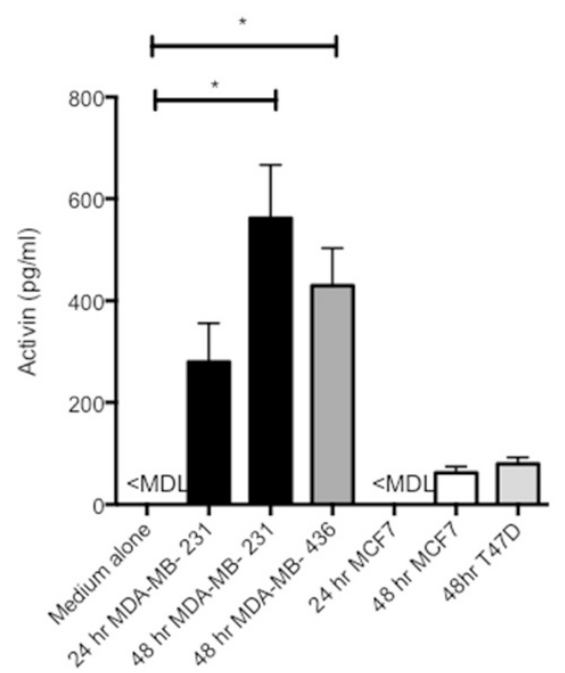

B

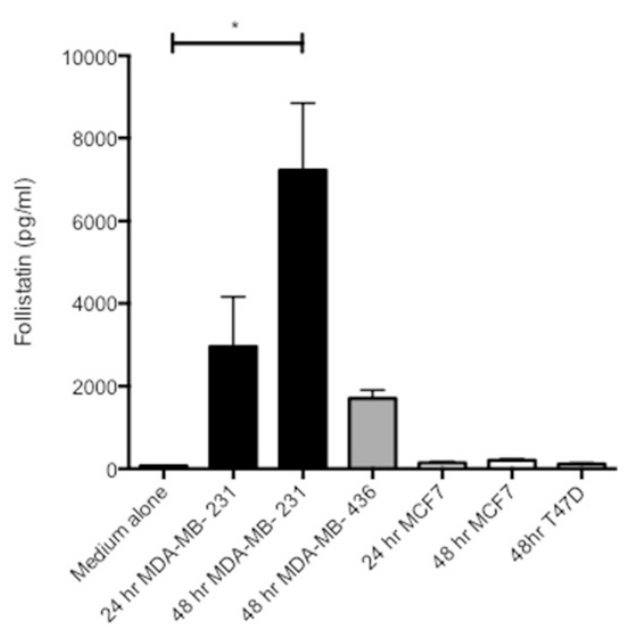

Figure 3 Activin and follistatin secretion from ER- breast cancer cell lines and ER + ve breast cancer cell lines. $1 \times 10^{5} \mathrm{MDA}-\mathrm{MB}-231$ or

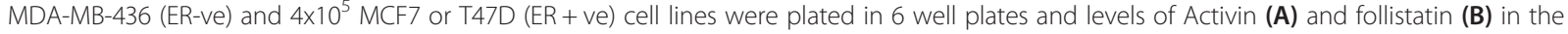
medium determined by ELISA at 24 and 48 hours. Data represents 3 replicates and 3 repeats. Mann Whitney test for significance comparing wells with cells to media alone (no cells), ${ }^{*} \mathrm{p}$ value $<0.05$. $<\mathrm{MDL}=$ below assay minimum detection limit.

(mean level pg/ml; MDA-MB-231 = 7224, MDA-MB$436=1704$ Figure 3B). These results suggest that ER-ve cells could be more dependent on activin A for regulation of cell growth than ER + ve cells, and may utilise secretion of follistatin as a mechanism of escape from the anti-proliferative effects of activin A. There is no clear evidence to indicate why tumor cells secrete a growth inhibitor such as activin [16], suggesting it may be have alternative functions that the cells escape from by alternative mechanisms i.e. secretion of inhibitors or down regulation of receptors.

\section{Zoledronic acid differentially affects proliferation of breast cancer cell lines according to ER status}

To evaluate if zoledronic acid could affect proliferation of ER + ve and ER-ve breast cancer cell lines MDA-MB231, MDA-MB-436, T47D and MCF7 cells were treated with $50 \mu \mathrm{M}$ ZOL or medium control for 48 hours and live cell count performed with trypan blue. ZOL significantly inhibited proliferation of both ER-ve cell lines compared to control (Cell count $\times 10^{5}$ MDA-MB-231 control 3.4 [SEM 0.79], ZOL 1.6 [SEM 0.25] p value 0.0009, MDA-MB-436 control 6.9 [SEM 0.33] ZOL 5.3 [SEM 0.14 ] $\mathrm{p}$ value 0.0043 ), but did not significantly alter proliferation of the ER + ve cell lines MCF7 and T47D (Figure 4).

\section{Zoledronic acid decreases follistatin secretion from ER-ve cell lines only}

To investigate if ZOL could affect the secretion of activin $\mathrm{A}$ and/or follistatin from MDA-MB-231 and MCF7 cells, both cell lines were exposed to medium $+/-25 \mu \mathrm{M} /$ $50 \mu \mathrm{M}$ ZOL for 48 hours. Activin A secretion was unaffected by ZOL in either cell line. In contrast, follistatin secretion was significantly decreased in MDA-MB-231 cells after exposure to ZOL (control $23378 \mathrm{pg} / \mathrm{ml}$ [SEM 5259], ZOL $9987 \mathrm{pg} / \mathrm{ml}$ [SEM 2871], p value $=0.0012$ ), and also fell in MDA-MB-436 cells (control $1928 \mathrm{pg} / \mathrm{ml}$ [SEM 188], ZOL 1592 pg/ml [SEM 65] p value 0.07), but did not change in MCF7 (Figure 5) or T47D cells (data not shown). We hypothesise that the biological activity of activin A depends on the ratio of activin A to follistatin in the tumour microenvironment. The literature reports a 4:1 molar ratio of follistatin:activin would neutralize activin [17]. ZOL reduced the molar ratio of follistatin:activin secreted by MDA-MB-231 cells (control ratio 14:1, ZOL ratio 4:1) (Figure 5B), compared to minimal change in MCF7 cells (control ratio 3:1, ZOL ratio 4:1) (Figure 5D) indicating ZOL has a more noticeable effect on the follistatin:activin ratio in ER-ve cell lines.

To evaluate the effects of a short (clinically achievable) exposure of ZOL on follistatin secretion in ER-ve cell lines, MDA-MB-231 and MDA-MB-436 cells were treated with $50 \mu \mathrm{M}$ ZOL for 4 hours, followed by 44 hours incubation in drug-free medium. The secretion of follistatin was significantly decreased in both cell lines by a 4-hour pulse of ZOL (mean follistatin pg/ml, MDA-MB-231; control $=17551 \quad$ [SEM 847], ZOL $=6106 \quad$ [SEM 1315] $\mathrm{p}$ value $=0.0015$. MDA-MB-436; control $=3209$ [SEM 236], $\mathrm{ZOL}=1667$ [SEM 116] p value $=0.001$ ) (Figure 6A). These data show that even a short exposure to ZOL decreases follistatin secretion from ER-ve cell lines. 


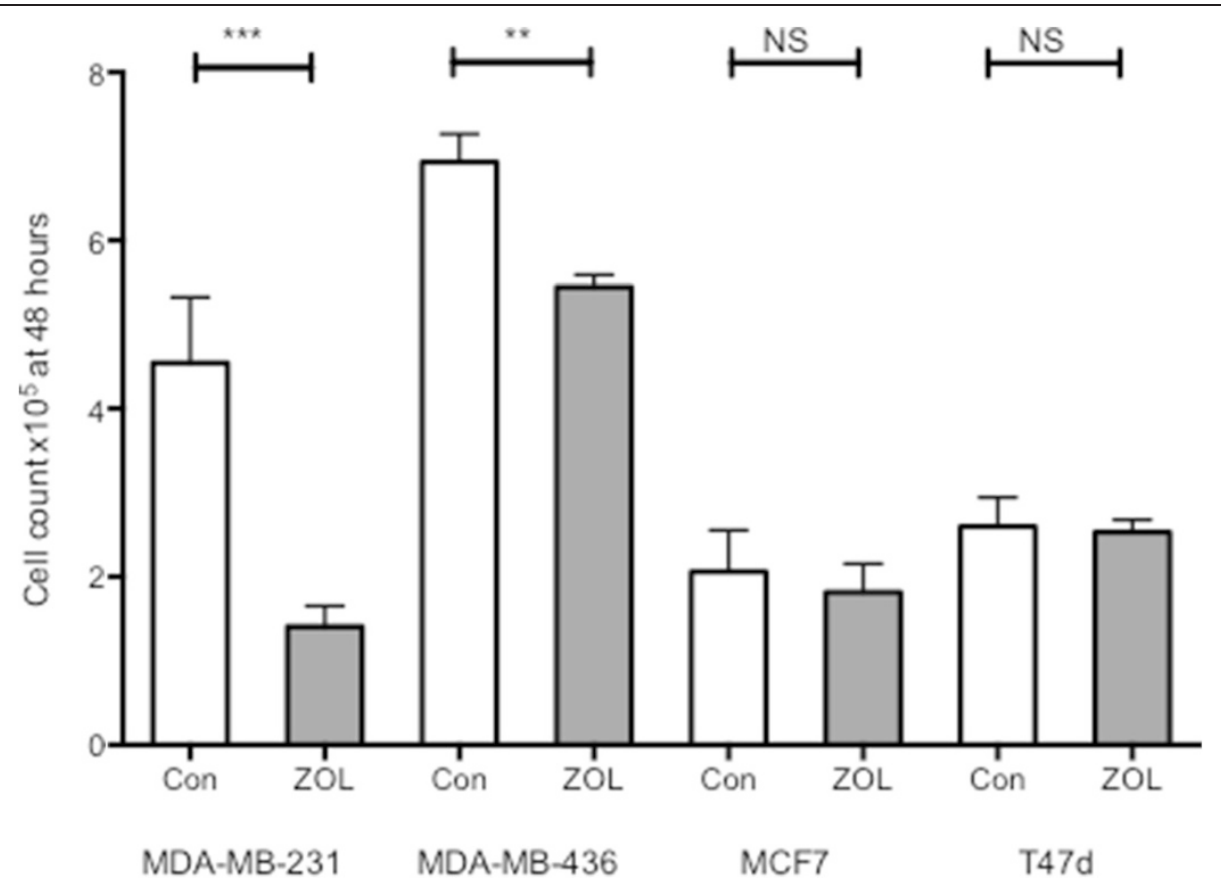

Figure 4 Effects of zoledronic acid on proliferation of ER + ve and ER-ve cell lines. 1×105 MDA-MB-231, MDA-MB-436 (ER-ve),MCF7 or T47D $(E R+v e)$ cell lines were plated in 6 well plates. Cell were treated for 48 hours with medium $+/-50 \mu \mathrm{M}$ ZOL. At 48 hours viable cell count was performed using trypan blue. Data represents 3 replicates and 3 repeats. Mann Whitney test for significance comparing control with ZOL treated, NS not significant, ${ }^{* *} p$ value $<0.005,{ }^{* * *} p$ value $<0.0005$.

Both ER + ve and ER-ve cell lines take up ZOL in vitro

ZOL increases accumulation of unprenylated small GTPases i.e. Rap1a via inhibition of the mevalonate pathway [18]. The lack of effect of ZOL on follistatin secretion in the ER + ve cells was considered to possibly reflect a limited drug uptake. To evaluate if the ER + ve and ER-ve cell lines used in this study had a similar levels of ZOL uptake we used western blotting to compare the accumulation of unprenylated Rapla (uRapla, a surrogate marker of ZOL uptake) in MCF7 and MDA-MB-231 cells treated with $\mathrm{ZOL}$, and if addition of the mevalonate pathway intermediary, geranylgeraniol $(\mathrm{GGOH})$, could inhibit the accumulation of uRapla. Both cell lines had increased levels of uRap1a in response to treatment with ZOL that was partially reversed by addition of GGOH (Figure 6B). These data suggest that the difference in follistatin secretion between ER-ve and ER + ve cell lines is not due differential cellular uptake of ZOL.

\section{Zoledronic acid reduces intracellular $\mathrm{C}$ terminus phosphorylated Smad2}

To evaluate if the activin-signaling pathway downstream of surface receptors is affected by $\mathrm{ZOL}$, localization and intracellular quantity of pSmad2C in MDA-MB-231 and MCF7 cells was assessed. Using an immunofluorescence method, we detected no significant difference in the percentage of cells with nuclear localization of $\mathrm{pSmad} 2 \mathrm{C}$ after treatment with ZOL compared to control in either cell line (Figure 7A-C). However, MDA-MB-231 cells exposed to CM from ZOL treated cells (containing low levels of follistatin) showed significantly higher levels of pSmad2C than cells exposed to CM from medium only treated cells (control $=0.29$ [SEM 0.065], $\mathrm{ZOL}=0.7$ [SEM 0.14], p value 0.0286) (Figure 7D). This effect was not seen in MDA-MB-231 cells exposed directly to $50 \mu \mathrm{M} Z \mathrm{ZOL}$, indicating that the decreased follistatin levels in CM from ZOL treated cells was responsible for the increase in intracellular levels of pSmad2C.

\section{Zoledronic acid decreases nuclear localization of linker phosphorylated Smad2}

Whereas pSmad2C is recognized to function as a tumour suppressor in breast cancer [19], pSmad2L may act as a tumour growth promoter [10]. We evaluated if ZOL could affect cellular localization of pSmad2L in MDA-MB-231 and MCF7 cells using immunofluorescence. The percentage of MDA-MB-231 cells with nuclear localization of pSmad2L was significantly decreased after treatment with $\mathrm{ZOL}$ (control $=50 \%$ [SEM 4.6], ZOL $=6.6 \%$ [SEM 1.1], $\mathrm{p}$ value $<0.0001)$. No significant difference was seen between ZOL and control in the MCF7 cells (Figure 7E-G). Using western blotting we found that ZOL did not cause a significant alteration in the total cellular levels of pSmad2L, suggesting that ZOL alters cellular localization of $\mathrm{pSmad} 2 \mathrm{~L}$ in MDA-MB-231, but not the total quantity (Figure 7H). 
A

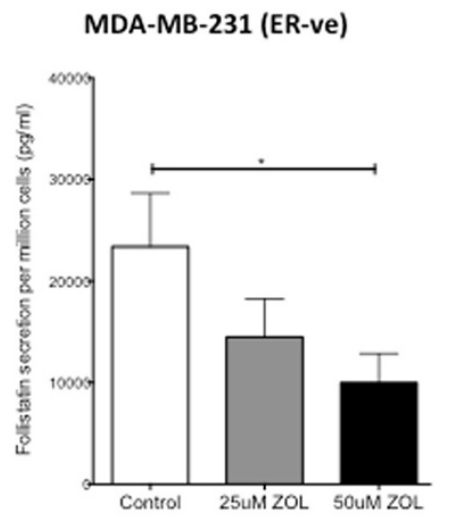

C

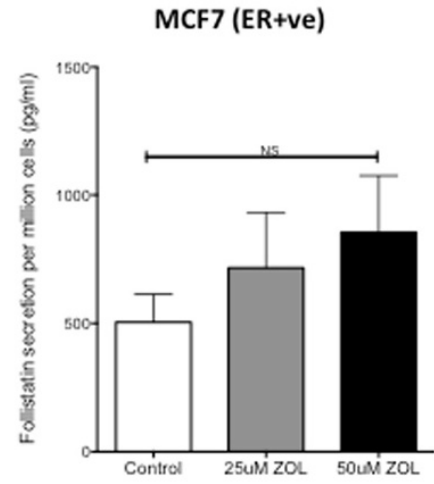

B

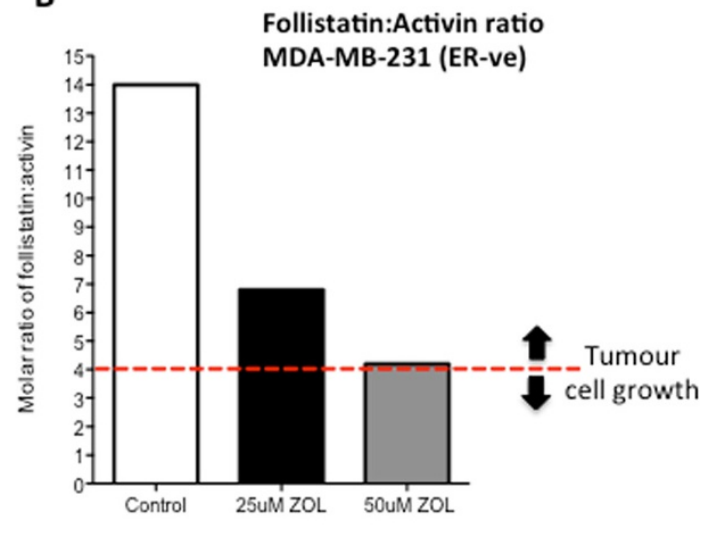

D Follistatin:Activin ratio MCF7 (ER+ve)

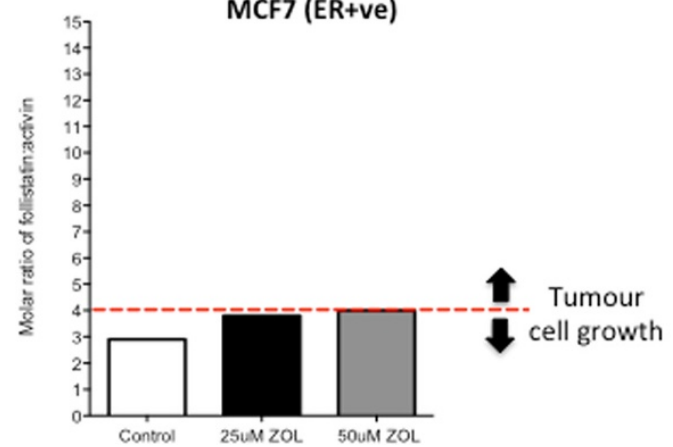

Figure 5 Effects of zoledronic acid on follistatin secretion and follistatin:activin ratio. MDA-MB-231 (A) and MCF7 (C) cells were treated with medium alone, $25 \mu \mathrm{M}$ or $50 \mu \mathrm{M}$ ZOL for 48 hours and levels of secreted activin and follistatin measured by ELISA. Molar ratio of follistatin: activin (B + D) was calculated by converting mean quantity of secreted protein per million cells $(\mathrm{pg} / \mathrm{ml})$ to $\mathrm{pmol} / \mathrm{l}$ by dividing by the molecular weight of each protein, and then expressed as a ratio. A molar ratio of 4 (dashed line) represents the level at which activin is neutralised by follistatin: an excess of follistatin:activin increases tumour growth (above dashed line). Data represents mean + SEM of 3 replicates and 3 repeats, ${ }^{*}=p$ value $<0.05$, NS not significant.
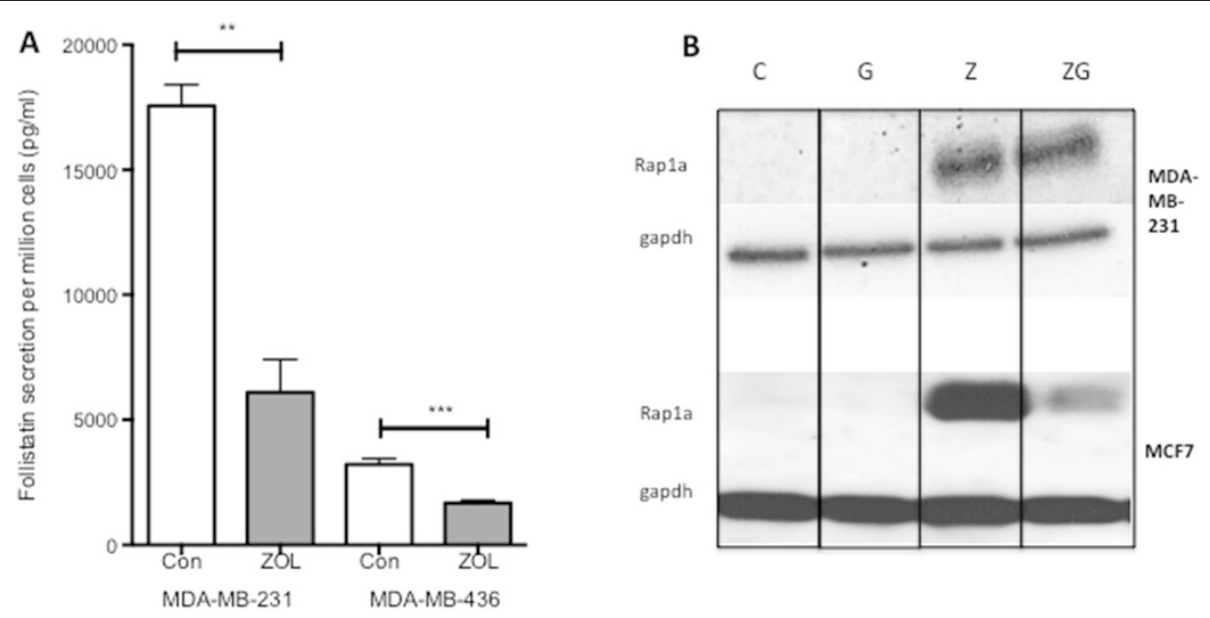

Figure 6 Effects of pulsed zoledronic acid on follistatin secretion from ER-ve cell lines. The difference in follistatin secretion according to ER status is not due to differences in cellular uptake of the drug. A. MDA-MB-231 and MDA-MB-436 cells were treated for 4 hours with $50 \mu \mathrm{M}$ of $\mathrm{ZOL}$ followed by a 44 hour incubation with medium alone or medium alone for 48 hours (CON). Follistatin levels in the supernatant was removed and processed for ELISA. Data represents mean + SEM of 3 replicates and 3 repeats, ${ }^{* *}=p$ value $<0.01,{ }^{* * *}=p$ value $<0.001$. B. MDAMB-231 and MCF7 cells were treated for 48 hours with medium alone (C), GGOH $50 \mu \mathrm{M}(\mathrm{G})$, Zol $10 \mu \mathrm{M}(\mathrm{Z})$ or both $\mathrm{G}$ and Z in combination (ZG). Rap1a antibody (1:200) used to assess levels of unprelylated protein and GAPDH $(1: 20,000)$ used as loading control. 


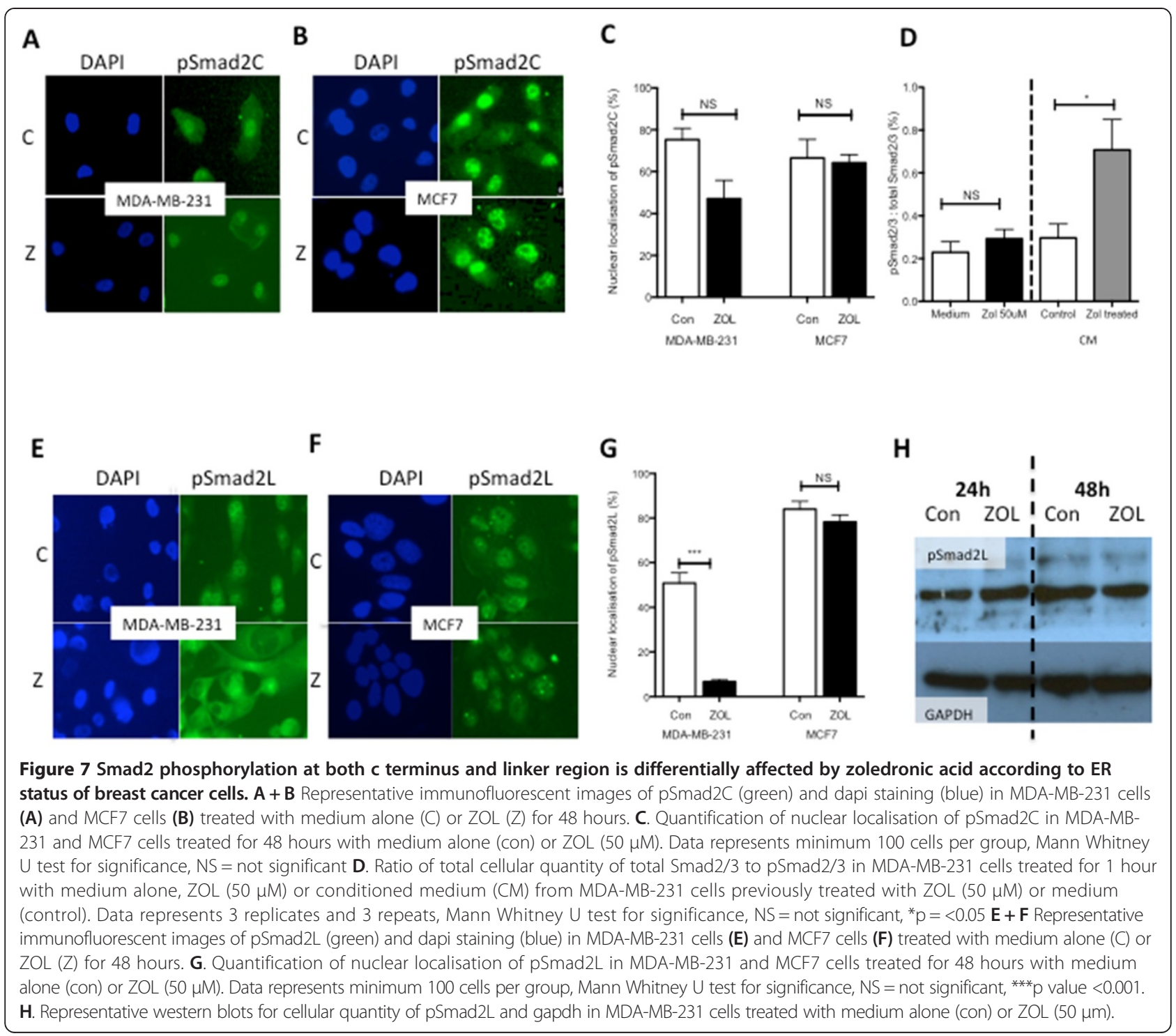

\section{Zoledronic acid decreases follistatin and PSmad2L expression in an ER-ve xenograft model}

In order to validate that $\mathrm{ZOL}$ induces changes in follistatin and pSmad2L in ER-ve tumours in vivo, ER-ve MDA-MB436 sub-cutaneous tumour sections from mice treated with or without ZOL $(100 \mu \mathrm{g} / \mathrm{kg}$, weekly for 6 weeks, equivalent to the $4 \mathrm{mg}$ clinical dose) were evaluated. Follistatin expression was scored for intensity and area of positive stain. Data were analysed using average scores from 2 assessors blinded to the treatment groups. No difference was seen in the intensity of follistatin staining, however, there was a significant decrease in the tumour area staining positive for follistatin in mice treated with ZOL compared to saline (Figure 8A-D). The number of cells with mitotic nuclei staining positive for pSmad2L was significantly lower in tumours from ZOL treated mice compared to saline treated (Figure 8E-F). These results suggest that using the dosing regime described, ZOL can directly alter expression of both follistatin and pSmad2L in ER-ve subcutaneous tumours in vivo.

\section{Discussion}

In this study we describe a novel anti-proliferative mechanism of action of ZOL in ER-ve breast cancer cells, involving the activin-signaling pathway, and suggest that this may contribute to the enhanced anti-tumour effect of ZOL in ER-ve breast cancers demonstrated in neoadjuvant clinical trials $[2,20]$.

In agreement with published data, we found that activin A inhibits proliferation of ER + ve MCF7 cells [21]. However, we saw a very similar inhibition of growth in ER-ve MDA-MB-231 cells, in contrast to previously reported data [10]. Kalkoven et al. suggested the mechanism responsible for resistance to the anti-proliferative effects 


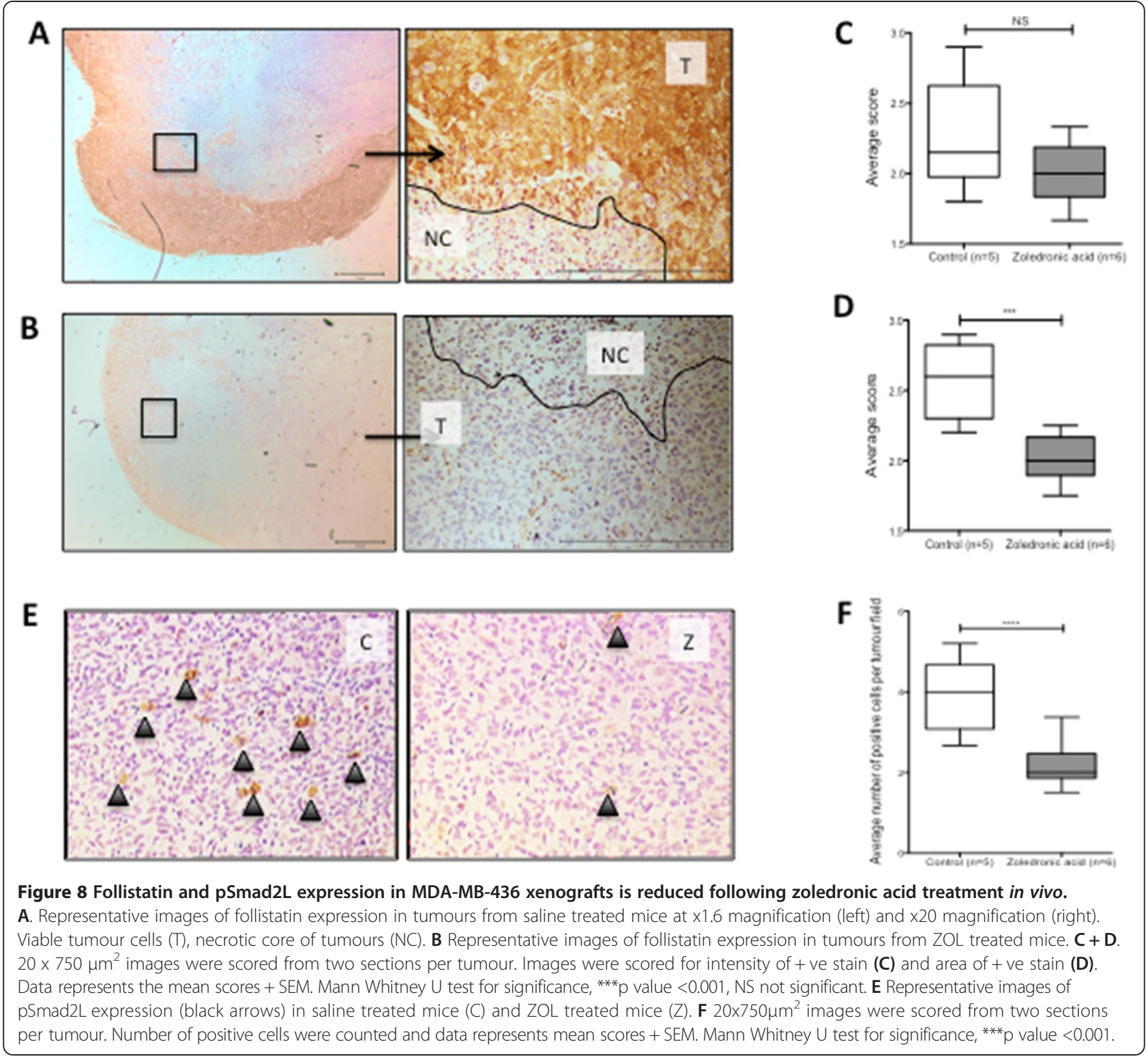

of activin A was located downstream of the receptor [11], however, alternative mechanisms such as the presence and/or effect of secreted activin neutralizers like follistatin was not evaluated.

Breast cancer cells have been shown to express the follistatin related gene (FLRG), encoding follistatin and follistatin related protein [12]. This same study also demonstrated that the anti-proliferative effect of activin was weak in MCF7 and undetectable in MDA-MB-436 cells. However, when endogenous secreted inhibitors i.e. follistatin were removed, Smad2C was phosphorylated in response to activin in both cell lines, and silencing of FLRG increased levels of pSmad2C and decreased proliferation in response to endogenous activin. These results support our data, demonstrating that activin inhibitors such as follistatin can neutralise the anti-proliferative effects of activin in both ER-ve and ER + ve cell lines.

We found that both activin A and follistatin were secreted from ER-ve and ER + ve cells, but at different levels, hence generating different effects on tumour cell proliferation. ER-ve cells secreted an excess of follistatin:activin, favouring cell proliferation, and in contrast to ER + ve cells which secreted an excess of activin:follistatin, favouring growth suppression. Previously published data have demonstrated that the activin $\beta$ A subunit is detected in higher levels in breast carcinoma compared to normal breast tissue [15], and activin type II cell surface receptors were attenuated with increasing tumour grade [22]. These studies did not include measurements of follistatin expression. It is possible that resistance to the tumour suppressive 
actions of activin in breast cancer is linked to the levels of secretion of activin neutralizing molecules such as follistatin, as well as a concurrent decrease in expression of activin type II receptors. This potential mechanism requires exploration in clinical neo-adjuvant breast cancer studies.

The differential effect of ZOL on follistatin secretion according to ER status of breast cancer cell lines in vitro and in vivo demonstrated in this study, has not been previously reported. However, other studies have shown a differential effect of ZOL on proliferation according to ER status. Rachner et al. demonstrated that MCF7 cells did not alter proliferation rates in response to $\mathrm{ZOL}$, whereas MDA-MB-231 cells showed a significant dosedependent inhibition of proliferation and increase in apoptosis via activation of caspase 3 and 7 [4]. We detected uRap1a in both MCF7 and MDA-MB-231 cells after treatment with ZOL, suggesting that variable uptake of the drug is not an explanation for the differing effects on follistatin. This is in agreement with a report by Monkkonen et al., showing accumulation of uRap1A and isopentenyl diphosphate (IPP) in both MCF7 and MDA-MB-436 cells following $24 \mathrm{~h}$ treatment with $25 \mu \mathrm{M}$ ZOL [23]. However, uptake of ${ }^{14} \mathrm{C}$-labelled ZOL is reported to be 3 fold lower in ER-ve BO2 cells compared to ER + ve T47D and MCF7 cells one hour after addition of $25 \mu \mathrm{M}$ ZOL [24]. These contrasting results may be due to the differences in dose and time of exposure to the drug. Whether the ZOLinduced reduction in follistatin secretion from ER-ve cells is due to a direct effect of the drug on the mevalonate pathway remains to be established.

We found that the decrease in follistatin secretion from MDA-MB-231 cells affected the downstream protein $\mathrm{Smad} 2$, increasing the levels of $\mathrm{pSmad} 2 / 3 \mathrm{C}$ relative to total Smad2/3. Phosphorylation of Smad2 at the $C$ terminus domain has been shown to suppress breast cancer cell invasion and metastases to bone in vivo. In a mouse model of bone metastasis, Smad2 knockdown in MDA-MB-231 cells resulted in significantly faster tumour establishment in bone compared to the parental cell line, suggesting a tumour suppressive role [25]. In clinical studies, a tissue microarray study of breast tumours from 426 patients showed that loss of $\mathrm{pSmad} 2 \mathrm{C}$ was associated with a shorter median overall survival (110.5 vs. 306.5 weeks, $\mathrm{p}=0.024)$, suggesting that this may be a tumour specific poor prognostic factor [19]. Moreover, phosphorylation of Smad2 at the linker region has been reported to alter its action from tumour suppression to tumour promotion. Phosphorylation at this site is primarily via cytoplasmic RAS and nuclear cyclin dependent kinases [10], as opposed to the canonical receptor-mediated activin pathway leading to $\mathrm{C}$ terminus phosphorylation. Small GTPases have been found to affect the activin signaling pathway, with Rap2 increasing activin cell surface receptor expression, potentially increasing cellular responses to endogenous and exogenous activin [26]. However, RAC1 has been shown to inhibit $\operatorname{smad} 2 / 3$ activation [27], suggesting small GTPases can have differential effects on the activin pathway. We showed that ZOL decreased nuclear localization of pSmad2L in ER-ve breast cancer cells in vitro and the number of cells staining positive in vivo. Whether this effect of $\mathrm{ZOL}$ is via a small GTPase/ RAS dependent mechanism remains to be confirmed, but ZOL has been shown to decrease RAS expression and activity in ER-ve cell lines (MDA-MB-231 and BRC-230), with inhibition of cell proliferation [28].

\section{Conclusion}

Taken together, our data support a potential dual mechanism of action of ZOL on the activin signaling pathway in ER-ve breast cancer cells in vitro and in vivo; firstly via a decrease in follistatin secretion leading to an increase in the tumour suppressor pSmad2C, and secondly via a decrease in nuclear localization of the tumour promotor pSmad2L. These data provide a possible novel direct anti-proliferative mechanism of action of ZOL on breast cancer cells involving activin signaling, that could contribute to the enhanced anti-tumour effects of the drug in neo-adjuvant clinical trials of patients with ER-ve breast cancer, and requires further research in clinical samples. The potential indirect anti-proliferative mechanism of action of ZOL on breast cancer cells in the bone microenvironment involving activin signaling also requires further investigation, and may contribute preclinical data to explain the results of clinical adjuvant bisphosphonate trials where the burden of residual disease is likely to be within niches such as the bone.

\section{Competing interests}

Professor Coleman declares remuneration for expert testimony from Novartis, all other authors declare they have no conflict of interest.

\section{Authors' contributions}

CW was responsible for data collection and writing of the manuscript. PDO was responsible for providing in vivo subcutaneous tumour samples. REC and $\mathrm{IH}$ were equally responsible for reviewing and critically appraising the manuscript and verifying the data interpretations and conclusions. All authors read and approved the final manuscript.

\section{Acknowledgements}

We are grateful for the support of Professor Simon Cross for his expert review of histology slides. This study was supported by a grant from Weston Park Hospital Cancer Charity, Sheffield Teaching Hospitals Charity and the Sunita Merali Trust, Sheffield, UK.

\section{Author details}

${ }^{1}$ Academic Unit of Clinical Oncology, University of Sheffield, Medical School, Sheffield, UK. ${ }^{2}$ Academic Department of Oncology, University of Sheffield, Medical School, Sheffield, UK.

Received: 29 September 2014 Accepted: 2 February 2015 Published online: 14 February 2015

\section{References}

1. Coleman RE, Winter MC, Cameron D, Bell R, Dodwell D, Keane M, et al. The effects of adding zoledronic acid to neoadjuvant chemotherapy on tumour 
response: exploratory evidence for direct anti-tumour activity in breast cancer. Br J Cancer. 2010;102(7):1099-105. doi:10.1038/sj.bjc.6605604.

2. Aft RL, Naughton M, Trinkaus K, Weilbaecher K. Effect of (Neo)adjuvant zoledronic acid on disease-free and overall survival in clinical stage I/II breast cancer. Br J Cancer. 2012;107(1):7-11. doi:10.1038/bjc.2012.210.

3. Horiguchi JHY, Miura D. A randomized controlled trial comparing zoledronic acid plus chemotherapy with chemotherapy alone as a neoadjuvant treatment in patients with HER2-negative primary breast cancer. J Clin Oncol. 2013;31:2013. suppl; abstr 1029.

4. Rachner TD, Singh SK, Schoppet M, Benad P, Bornhauser M, Ellenrieder V, et al. Zoledronic acid induces apoptosis and changes the TRAIL/OPG ratio in breast cancer cells. Cancer Lett. 2010;287(1):109-16. doi:10.1016/j.canlet.2009.06.003.

5. Gnant M, Clezardin P. Direct and indirect anticancer activity of bisphosphonates: a brief review of published literature. Cancer Treat Rev. 2012;38(5):407-15. doi:10.1016/j.ctrv.2011.09.003.

6. Winter MC, Wilson C, Syddall SP, Cross S, Evans A, Ingram C, et al. Neoadjuvant chemotherapy with or without zoledronic acid in early breast cancer-a randomized biomarker pilot study. Clin Cancer Res. 2013;19(10):2755-65. doi:10.1158/1078-0432.CCR-12-3235.

7. Wilson CWM, Coleman RE, Ottewell P, Evans AC, Holen I. Differential antitumour effects of zoledronic acid in breast cancer according to ER status and levels of female hormones. Miami, Florida: Cancer and Bone Society and the International Bone and Mineral Society; 2013

8. Bloise E, Couto HL, Massai L, Ciarmela P, Mencarelli M, Borges LE, et al. Differential expression of follistatin and FLRG in human breast proliferative disorders. BMC Cancer. 2009;9:320. doi:10.1186/1471-2407-9-320.

9. Liu T, Feng XH. Regulation of TGF-beta signalling by protein phosphatases. Biochem J. 2010;430(2):191-8. doi:10.1042/BJ20100427.

10. Matsuzaki K. Smad phosphoisoform signaling specificity: the right place at the right time. Carcinogenesis. 2011;32(11):1578-88. doi:10.1093/carcin/bgr172.

11. Kalkhoven E, Roelen BA, de Winter JP, Mummery CL, Van den Eijnden-Van Raaij AJ, Van der Saag PT, et al. Resistance to transforming growth factor beta and activin due to reduced receptor expression in human breast tumor cell lines. Cell Growth Differ. 1995;6(9):1151-61.

12. Razanajaona D, Joguet S, Ay AS, Treilleux I, Goddard-Leon S, Bartholin L, et al. Silencing of FLRG, an antagonist of activin, inhibits human breast tumor cell growth. Cancer Res. 2007;67(15):7223-9. doi:10.1158/0008-5472.CAN-07-0805.

13. Ottewell PD, Monkkonen $\mathrm{H}$, Jones M, Lefley DV, Coleman RE, Holen I. Antitumor effects of doxorubicin followed by zoledronic acid in a mouse model of breast cancer. J Natl Cancer Inst. 2008;100(16):1167-78. doi:10.1093/jnci/djn240.

14. Burdette JE, Jeruss JS, Kurley SJ, Lee EJ, Woodruff TK. Activin A mediates growth inhibition and cell cycle arrest through Smads in human breast cancer cells. Cancer Res. 2005;65(17):7968-75. doi:10.1158/0008-5472.CAN-04-3553.

15. Reis FM, Cobellis L, Tameirao LC, Anania G, Luisi S, Silva IS, et al. Serum and tissue expression of activin a in postmenopausal women with breast cancer. J Clin Endocrinol Metab. 2002;87(5):2277-82.

16. Reis FM, Luisi $S$, Carneiro MM, Cobellis L, Frederico M, Camargos AF, et al. Activin, inhibin and the human breast. Mol Cell Endocrinol. 2004;225(1-2):77-82. doi:10.1016/j.mce.2004.02.016.

17. Harrison CA, Chan KL, Robertson DM. Activin-A binds follistatin and type II receptors through overlapping binding sites: generation of mutants with isolated binding activities. Endocrinology. 2006;147(6):2744-53. doi:10.1210/en.2006-0131.

18. Luckman SP, Coxon FP, Ebetino FH, Russell RG, Rogers MJ. Heterocyclecontaining bisphosphonates cause apoptosis and inhibit bone resorption by preventing protein prenylation: evidence from structure-activity relationships in J774 macrophages. J Bone Miner Res. 1998;13(11):1668-78. doi:10.1359/jbmr.1998.13.11.1668.

19. Xie W, Mertens JC, Reiss DJ, Rimm DL, Camp RL, Haffty BG, et al. Alterations of Smad signaling in human breast carcinoma are associated with poor outcome: a tissue microarray study. Cancer Res. 2002;62(2):497-505.

20. Horiguchi J, Hasegawa Y, Miura D, Ishikawa T, Hayashi M, Takao S, et al. A randomized controlled trial comparing zoledronic acid plus chemotherapy with chemotherapy alone as a neoadjuvant treatment in patients with HER2negative primary breast cancer. J Clin Oncol. 2013;31:2013. suppl; abstr 1029.

21. Cocolakis E, Lemay S, Ali S, Lebrun J. The p38 MAPK pathway is required for cell growth inhibition of human breast cancer cells in response to activin. J Biol Chem. 2001;276(21):18430-6. doi:10.1074/jbc.M010768200.

22. Jeruss JS, Sturgis CD, Rademaker AW, Woodruff T. Down-regulation of activin, activin receptors, and Smads in high-grade breast cancer. Cancer Res. 2003;63(13):3783-90
23. Monkkonen H, Kuokkanen J, Holen I, Evans A, Lefley D, Jauhianen M, et al. Bisphosphonate-induced ATP analog formation and its effect on inhibition of cancer cell growth. Anticancer Drugs. 2008;19(4):391-9. doi:10.1097/CAD.0b013e3282f632bf.

24. Benzaid I, Monkkonen H, Stresing V, Bonnelye E, Green J, Monkkonen J, et al. High phosphoantigen levels in bisphosphonate-treated human breast tumors promote Vgamma9Vdelta2 T-cell chemotaxis and cytotoxicity in vivo. Cancer Res. 2011;71(13):4562-72. doi:10.1158/0008-5472.CAN-10-3862.

25. Petersen M, Pardali E, van der Horst G, Cheung H, Van den Hoogen C, Van der Pluijm G, et al. Smad2 and Smad3 have opposing roles in breast cancer bone metastasis by differentially affecting tumor angiogenesis. Oncogene. 2010;29(9):1351-61. doi:10.1038/onc.2009.426.

26. Choi SC, Kim GH, Lee SJ, Park E, Yeo CY, Han JK. Regulation of activin/nodal signaling by Rap2-directed receptor trafficking. Dev Cell. 2008;15(1):49-61. doi:10.1016/j.devcel.2008.05.004

27. Ungefroren H, Groth S, Sebens S, Lehnert H, Gieseler F, Fandrich F. Differential roles of Smad2 and Smad3 in the regulation of TGF-beta1-mediated growth inhibition and cell migration in pancreatic ductal adenocarcinoma cells: control by Rac1. Mol Cancer. 2011;10:67. doi:10.1186/1476-4598-10-67.

28. Ibrahim T, Mercatali L, Sacanna E, Tesei A, Carloni S, Ulivi P, et al. Inhibition of breast cancer cell proliferation in repeated and non-repeated treatment with zoledronic acid. Cancer Cell Int. 2012;12(1):48. doi:10.1186/1475-2867-12-48.

\section{Submit your next manuscript to BioMed Central and take full advantage of:}

- Convenient online submission

- Thorough peer review

- No space constraints or color figure charges

- Immediate publication on acceptance

- Inclusion in PubMed, CAS, Scopus and Google Scholar

- Research which is freely available for redistribution 